\title{
A redescription of ZaVRelia bragremia Guo \& WAng, 2007 (Diptera: Chironomidae)
}

\author{
Xiao-Long Lin ${ }^{1, *}$, Xin-Hua Wang ${ }^{2}$ \\ ${ }^{1}$ Department of Natural History, NTNU University Museum, Norwegian University of Science and \\ Technology,NO-7491,Trondheim, Norway.E-mail: lin880224@gmail.com \\ ${ }^{2}$ College of Life Sciences, Nankai University, 300071, Tianjin, China. E-mail: xhwang@nankai.edu.cn \\ *Corresponding author
}

\begin{abstract}
Examination of the holotype of Zavrelia bragremia Guo \& Wang, 2007, revealed a few mistakes in the original description. Based on the type material and additional adult males from Oriental China, Z. bragremia is here redescribed and figured. An updated identification key to the males of Zavrelia is given.
\end{abstract}

\section{Introduction}

The genus Zavrelia was erected by Kieffer, Thienemann and Bause in Bause (1913) with $Z$. pentatoma as the type species. The genus belongs to the subtribe Zavreliina within tribe Tanytarsini, subfamily Chironominae and was reviewed by Ekrem and Stur (2009). At present, there are eleven valid species: Z. pentatoma Kieffer \& Bause in Bause, 1913, Z. simantoneoa (Sasa, Suzuki \& Sakai, 1998), Z. tusimatijea (Sasa \& Suzuki, 1999), Z. clinovolsella Guo \& Wang, 2004, Z. bragremia Guo \& Wang, 2007, Z. elenae Zorina, 2008, Z. pseudopentatoma Zorina, 2008, Z. aristata Ekrem \& Stur, 2009, Z. casasi Ekrem \& Stur, 2009, Z. hudsoni Ekrem \& Stur, 2009 and Z. sinica Ekrem \& Stur, 2009 (Ekrem and Stur 2009, Kobayashi 2014). Only four species of the genus Zavrelia have previously been recorded in China: Z. clinovolsella, Z. bragremia, Z. pentatoma and $Z$. sinica (Ekrem and Stur 2009). The known immature stages of most Zavrelia species occur in streams and rivers; except $Z$. pentatoma which is found in standing waters.

Here, we redescribe Z. bragremia based on the holotype and additional recently collected specimens, and give an updated identification key to the adult males of Zavrelia.

\section{Material and Methods}

Field work was conducted in China to supplement material for Z. bragremia. Studied specimens were slide-mounted in Euparal, following the proce- dures outlined by Sæther (1969). Morphological terminology follows Sæther (1980). Colouration is based on slide-mounted specimens. Measurements are given as ranges followed by the mean, when four or more specimens are measured, followed by the number of specimens measured (n) in parentheses. Digital photographs were taken with a resolution of 300 dpi using a Leica DFC420 camera mounted on a Leica DM6000 B compound microscope using bright field setting and the software Leica Application Suite 4.8. The holotype is deposited in the College of Life Sciences, Nankai University, Tianjin, China (BDN); several examined specimens are deposited in the NTNU University Museum (NTNU-VM).

\section{Zavrelia bragremia Guo \& Wang, 2007}

(Figs 1-10)

Zavrelia bragremia Guo \& Wang, 2007: 318, figs 1-3. Holotype $\widehat{\sigma}$ (BDN: 5486), China, Sichuan Province, Emeishan City, $29.60^{\circ} \mathrm{N}, 103.48^{\circ} \mathrm{E}$, 17.v.1986, XH Wang.

Additional examined specimens. $8 \hat{\partial} \widehat{\partial}$ (BDN: G5A49, G5A53-56, G5A69; NTNU-VM: G5A4, G5A7), China, Zhejiang Province, Jinhua City, Pan'an County, Dapanshan National Nature Reserve, Huaxi Village, near a stream, $28.792^{\circ} \mathrm{N}$, $120.525^{\circ} \mathrm{E}, 800 \mathrm{~m}$ a.s.1., $17-21$.vii.2012, light trap, XL Lin.

\section{Diagnosis}

The adult male of Zavrelia bragremia can be distinguished from known species of Zavrelia by the following combination of characters: wing length 0.88-1.04 mm; AR 0.91-1.08; frontal tubercle minute; $\mathrm{LR}_{1}$ about 2.09; laterosternite without seta; anal point with small spinulae between the anal crests at base, but without microtrichia; setiger of superior volsella without conspicuous constriction in apical $1 / 3$. 


\section{Description}

Adult male ( $\mathrm{n}=5$, unless otherwise stated). Total length $1.24-1.34,1.29 \mathrm{~mm}$. Wing length 0.88-1.04, $0.95 \mathrm{~mm}$. Total length/wing length $1.31-1.52,1.38$.

Colouration. Head capsule and mouthparts light brown, antenna and eyes dark brown to black. Thorax ground colour brown with dark brown stripes anteriorly on scutum, laterally under parapsidal suture, postnotum and on preepisternum, scutellum paler; legs and abdomen brown.

Head (Figs 1-2). Antenna (Fig. 1) with 10 flagellomeres, ultimate flagellomere 260-295, 278 $\mu \mathrm{m}$ long. AR 0.91-1.08, 0.98. Frontal tubercles minute, 3-4, $3 \mu \mathrm{m}$ long, 3-4, $3 \mu \mathrm{m}$ wide at base. Eye (Fig. 2) hairy, reniform, without dorsomedial extension. Temporal setae 5-6, 5. Clypeus with 8-10, 9 setae. Tentorium 88-98, $93 \mu \mathrm{m}$ long, 8-15, $12 \mu \mathrm{m}$ wide. Palpomere lengths (in $\mu \mathrm{m}$ ): $20-27$, 23 ; 25-30, 27; 65-75, 68; 78-83, 81; 110-120,
115. Third palpomere with one sensillum clavatum distally.

Thorax chaetotaxy (Fig. 3). Acrostichals $6(n=4)$; dorsocentrals 5-6, 6; humerals 0 ; prealars 1-2, 1; scuttellars 4; haltere with 3-6, 4 setae.

Wing (Fig. 4). VR 1.21-1.35, 1.28. Brachiolum with one seta, Sc bare, R with 17-25, 20 setae, $\mathrm{R}_{1}$ with 16-20, 17 setae, $\mathrm{R}_{4+5}$ with 15-20, 17 setae, $\mathrm{M}_{1+2}$ with 40-53, 47 setae, $\mathrm{M}_{3+4}$ with 14-18, 16 setae, false vein with 78-86, 81 setae, $\mathrm{Cu}$ with 14-17, 16 setae, $\mathrm{Cu}_{1}$ with 10-12, 11 setae, $\mathrm{PCu}$ with 36-43, 40 setae, An with 17-18, 18 setae, remaining veins bare. Cell $\mathrm{r}_{4+5}$ with c. 190-210, 201 setae, $m$ with 3-8, 5 setae, $m_{1+2}$ with c. 180-200, 190 setae, $\mathrm{m}_{3+4}$ with $62-80,71$ setae, cu+an with 85-100, 91 setae, remaining cells bare. Anal lobe strongly reduced.

Legs. Fore leg bearing single tibial spur, 10-18, 14 $\mu \mathrm{m}$ long. Combs of mid tibia 13-18, $15 \mu \mathrm{m}$ wide with 13-15, $14 \mu \mathrm{m}$ long spur, and 11-13, $12 \mu \mathrm{m}$

Table 1. Lengths (in $\mu \mathrm{m}$ ) and proportions of legs for Zavrelia bragremia Guo \& Wang, adult male.

\begin{tabular}{lllllll}
\hline & $\mathbf{f e}$ & $\mathbf{t i}$ & $\mathbf{t a}_{\mathbf{1}}$ & $\mathbf{t a}_{\mathbf{2}}$ & $\mathbf{t a}_{\mathbf{3}}$ & $\mathbf{t a}_{\mathbf{4}}$ \\
\hline $\mathrm{p}_{1}$ & $309-490,435$ & $220-250,235$ & $460(\mathrm{n}=1)$ & $240(\mathrm{n}=1)$ & $190(\mathrm{n}=1)$ & $150(\mathrm{n}=1)$ \\
$\mathrm{p}_{2}$ & $410-480,458$ & $320-360,340$ & $220-240,225$ & $85-100,90$ & $50-70,57$ & $30-50,45$ \\
$\mathrm{p}_{3}$ & $470-560,512$ & $360-400,388$ & $240-300,270$ & $145-170,150$ & $120-150,136$ & $70-90,80$ \\
\hline & $\mathbf{t a}_{\mathbf{5}}$ & $\mathbf{L R}$ & $\mathbf{B V}$ & $\mathbf{S V}$ & $\mathbf{B R}$ & \\
\hline $\mathrm{p}_{1}$ & $70(\mathrm{n}=1)$ & $2.09(\mathrm{n}=1)$ & $1.65(\mathrm{n}=1)$ & $1.33(\mathrm{n}=1)$ & $3.76(\mathrm{n}=1)$ \\
$\mathrm{p}_{2}$ & $25-43,34$ & $0.64-0.70,0.67$ & $4.13-5.18,4.52$ & $3.24-3.70,3.51$ & $2.67-5.40,3.92$ \\
$\mathrm{p}_{3}$ & $50(\mathrm{n}=4)$ & $0.66-0.72,0.69$ & $2.77-2.83,2.79$ & $3.25-3.46,3.32$ & $3.86-5.33,4.45$ \\
\hline
\end{tabular}

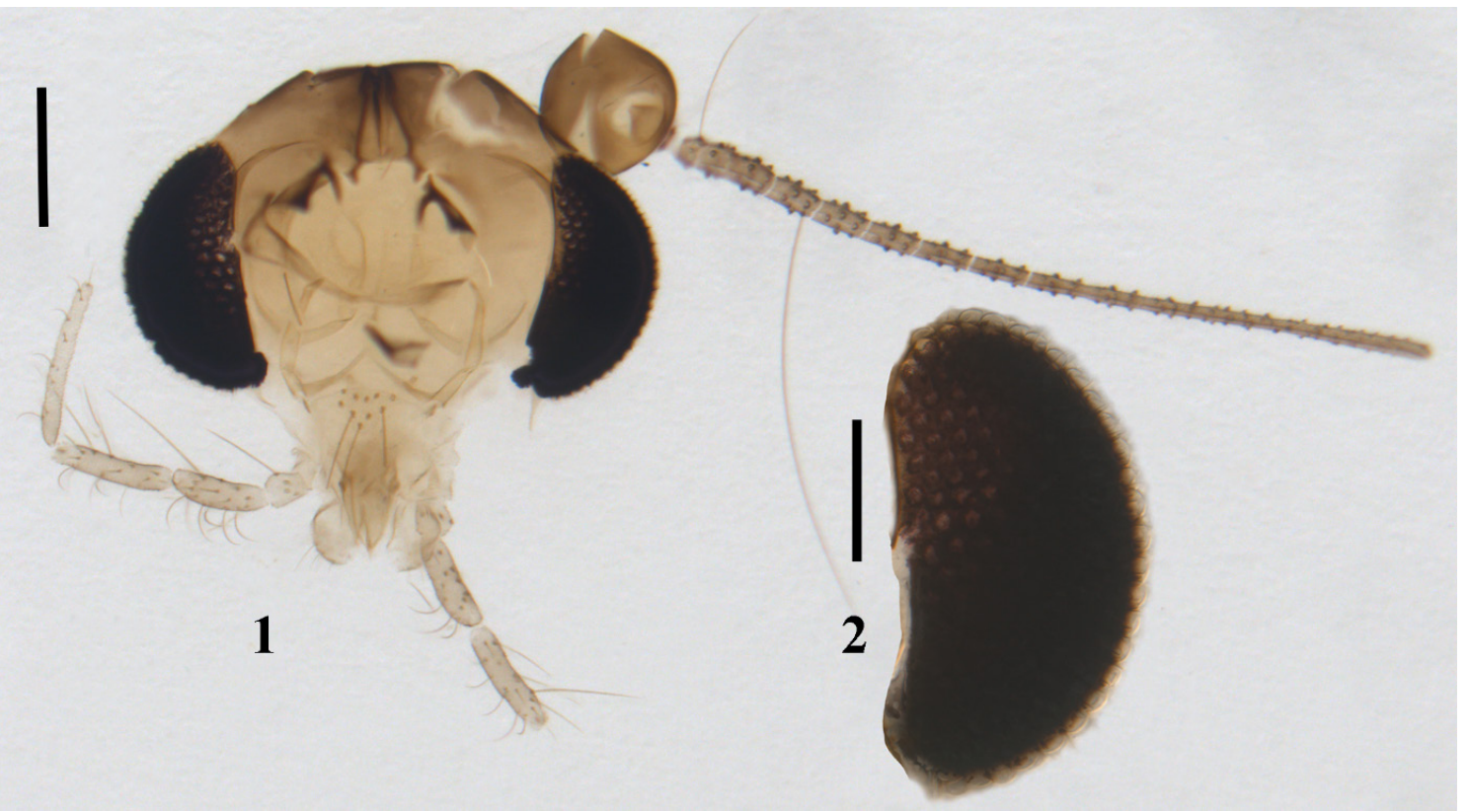

Figures 1-2. Zavrelia bragremia Guo \& Wang, 2007, male. 1, head and antenna, scale $=100 \mu \mathrm{m}$; 2, eye, scale $=50 \mu \mathrm{m}$. 

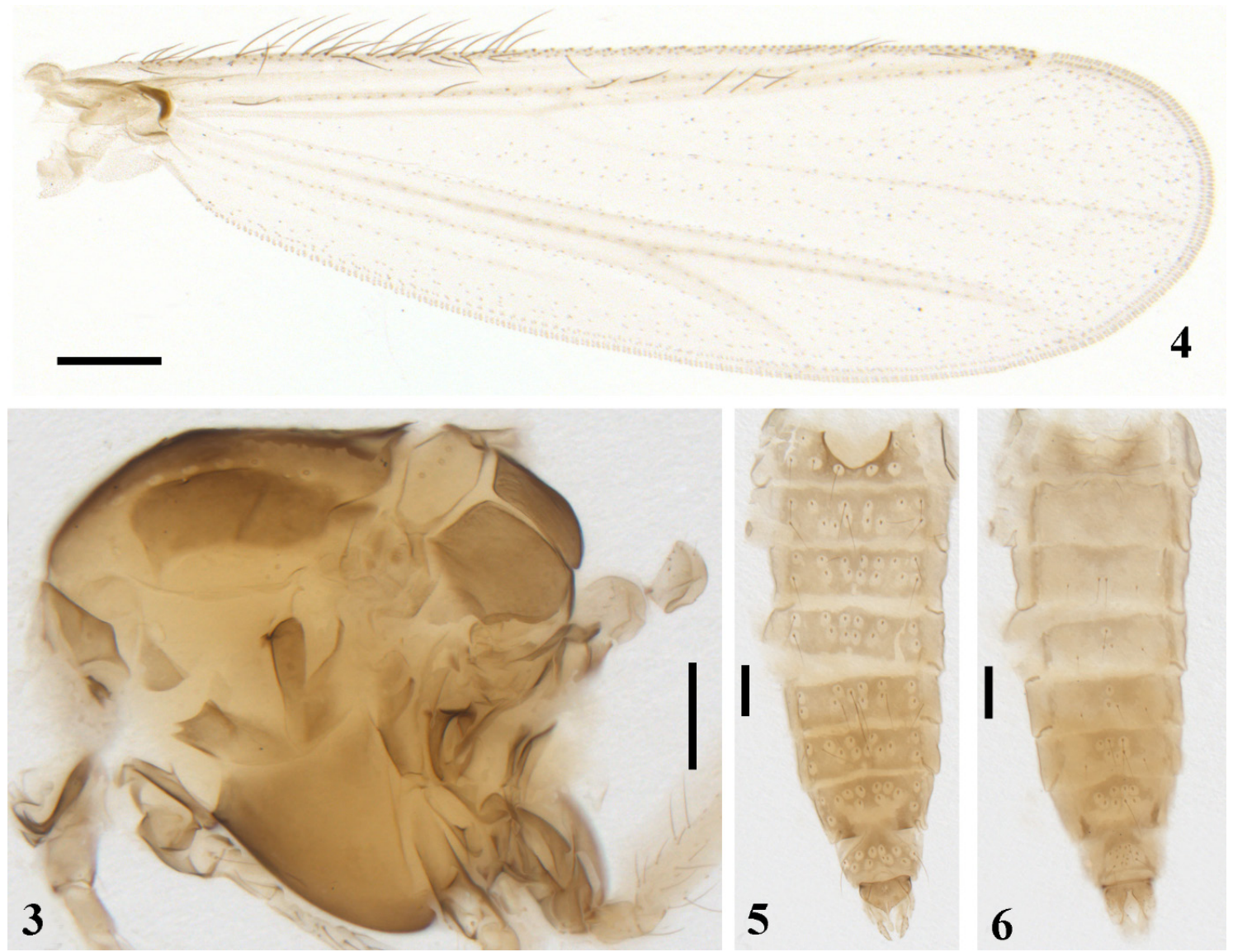

Figures 3-6. Zavrelia bragremia Guo \& Wang, 2007, male. 3, thorax; 4, wing; 5, abdomen, dorsal view; 6, abdomen, ventral view. Scales $=100 \mu \mathrm{m}$.

wide with 10-13, $12 \mu \mathrm{m}$ long spur; combs of hind tibia $15-20,17 \mu \mathrm{m}$ wide with $15-20,18 \mu \mathrm{m}$ long spur, $15-18,17 \mu \mathrm{m}$ wide with $10-15,13 \mu \mathrm{m}$ long spur. Sensilla chaetica on basitarsus of mid leg absent. Lengths (in $\mu \mathrm{m}$ ) and proportions of legs as in Table 1.

Abdomen (Figs 5-6). Setation see Figs 5-6.

Hypopygium (Figs 7-10). Laterosternite IX without seta. Anal tergite 56-68, $62 \mu \mathrm{m}$ long, with 3-7, 5 median setae, 16-20, 18 apical setae. Anal point well developed, 17-20, $19 \mu \mathrm{m}$ long, with 17-30, 23 small spinules scattered between anal crests at base, apex rounded; no microtrichia free area around base of anal point. Transverse sternapodeme $30-41,37 \mu \mathrm{m}$ long, without oral projections. Phallapodeme 45-75, $59 \mu \mathrm{m}$ long. Superior volsella broadly digitiform with pointed apex, bearing two anteromedian and 3-5, 4 dorsal setae; microtrichia apparently absent. Median volsella (Fig. 10) short, knob-shaped, with 3-4, 4 medially directed simple and subulate lamellae. Inferior volsella slightly curved, $45-55,51 \mu \mathrm{m}$ long, bearing $6-9,7$ setae in distal part. Gonocoxite $65-73,70$ $\mu \mathrm{m}$ long. Gonostylus $38-45,41 \mu \mathrm{m}$ long, curved inwards, broadest at middle, tapering to narrowly rounded apex. HR 1.55-1.83, 1.67, HV 2.62-3.35, 3.03 .

Immature stages and adult females. Unknown.

\section{Identification key to adult males of Zavrelia}

1. Anal point densely covered with strong spines Z. pentatoma

- Anal point bare, with microtrichia or short spinules only

2

2. Setiger of superior volsella with conspicuous constriction in apical $1 / 3$

- Setiger of superior volsella without conspicuous constriction in apical $1 / 3$ 5

3. Setiger of superior volsella with pointed apex .. Z. clinovolsella

- Setiger of superior volsella with somewhat rectangular apex 4

4. Anal point with only few microtrichia in between crests; distinct microtrichia-free areas on anal tergite around base of anal point ...... Z. sinica 
- Anal point with numerous microtrichia in between crests; microtrichia present all around base of anal point

Z.pseudopentatoma

5. Anal point with small spinules in between crests

- Anal point bare, or with microtrichia in between crests at most

6. AR 1.23; wing length about $1.50 \mathrm{~mm}$; $\mathrm{LR}_{1}$ about 1.45; laterosternite with one seta; anal point with small spinules scattered between entire length of anal crests

Z. tusimatijea

- AR 0.91-1.08; wing length 0.88-1.04 mm; LR $_{1}$ about 2.09; laterosternite without seta; anal point with small spinules scattered between anal crests at anal point base only Z. bragremia

7. Anal point bare 8

-Anal point with microtrichia in between crests . 9

8. AR 1.00-1.18; LR 1.36-1.46; superior volsella with pointed apex Z. elenae

- AR 0.45; LR 1 1.96; superior volsella with rounded apex

Z. simantoneoa

9. Wing length c. $1.40 \mathrm{~mm}$; AR c. 0.75.. Z. hudsoni -Wing length c. $1.00 \mathrm{~mm}$; AR c. $0.90 \ldots$ Z. aristata
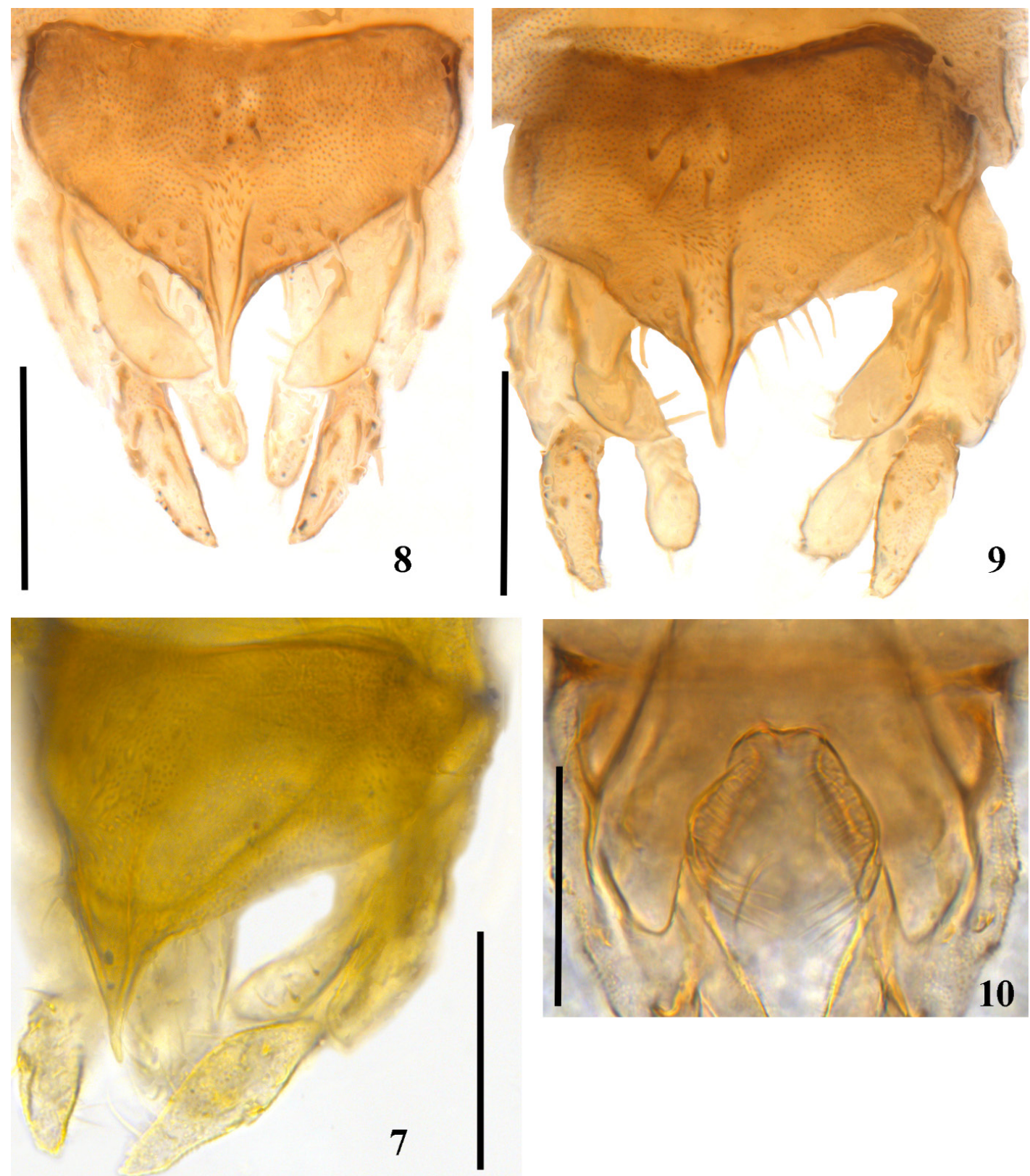

Figures 7-10. Zavrelia bragremia Guo \& Wang, 2007, male. 7, holotype hypopygium, dorsal view; 8, hypopygium (BDN: G5A69), dorsal view; 9, hypopygium (BDN: G5A53), dorsal view; 10, median volsellae. Scales $=50 \mu \mathrm{m}$. 


\section{Discussion}

Zavrelia bragremia was described based on a single specimen from Sichuan, China by Guo and Wang (2007). By reexamining the holotype, we found that some diagnostic characters in the original description require emendation. For instance, the anal tergite bears three to seven median setae; the anal point does not have two spines (an artifact resulting from slide preparation might have been interpreted as spines, Fig. 7); the anal point has scattered spinules between anal crests at anal point base only. The only complete recently collected specimen of $Z$. bragremia from Oriental China has also a higher $\mathrm{LR}_{1}$ value (2.09) - the highest within all known Zavrelia. This was previously unknown since the fore tarsi are missing in the holotype.

Due to slide preparation methods and a distinct variability of Zavrelia species, it is extremely difficult to observe and define key characters to accurately separate species on the basis of their morphology. An intraspecific variability of the type species Zavrelia pentatoma was detected by Giłka (2008). On the other hand, some morphological variable characters of $Z$. pentatoma have been found as diagnostic in other species like the presence of an obvious constriction in the apical $1 / 3$ of the setiger of the superior volsella (see identification key). Cryptic species, particularly within some of the more widely distributed Zavrelia are thus expected to be revealed. A complete DNA barcode reference library of Zavrelia would be beneficial to explore the species boundaries within this genus, since DNA barcoding (Hebert et al. $2003 \mathrm{a}, \mathrm{b})$ has proven effective in biodiversity assessments and taxonomic revisions (e.g. Anderson et al. 2013, Lin et al. 2017). At present (August 2017), however, there is only one species, Zavrelia pentatoma, with DNA barcodes in the Barcode of Life Data Systems (BOLD, http://www.boldsystems.org/).

\section{Acknowledgements}

We are indebted to Prof. Torbjørn Ekrem for his revision on the manuscript, and the two anonymous reviewers for their comments. We are also indebted to Su-Jiong Zhang for his help in field work in the Dapanshan National Nature Reserve, Jinhua, Zhejiang, China.

\section{References}

Anderson, A.M., Stur, E. and Ekrem, T. 2013. Molecular and morphological methods reveal cryptic diversity and three new species of Nearctic Micropsectra (Diptera: Chironomi- dae). - Freshwater Science 32: 892-921. DOI: http://dx.doi.org/10.1899/12-026.1.

Bause, E. 1913. Die Metamorphose der Gattung Tanytarsus und einiger verwandter Tendipedidenarten. Ein Beitrag zur Systematik der Tendipediden. - Archiv für Hydrobiologie Supplement 2: 1-126.

Ekrem, T. and Stur, E. 2009. A review of the genus Zavrelia (Diptera: Chironomidae). - European Journal of Entomology 106: 119-144. DOI: http://dx.doi.org/10.14411/eje.2009.016.

Giłka, W. 2008. An intraspecific morphological variability of Zavrelia pentatoma Kieffer (Diptera: Chironomidae). - Dipteron, Bulletin of the Dipterological Section of the Polish Entomological Society 24: 11-15.

Guo, Y.H. and Wang, X.H. 2007. Zavrelia bragremia sp. nov. from China (Diptera, Chironomidae, Tanytarsini). - Acta Zootaxonomica Sinica 32: 318-320.

Hebert, P.D.N., Cywinska, A. and Ball, S.L. 2003a. Biological identifications through DNA barcodes. - Proceedings of the Royal Society of London B: Biological Sciences 270: 313-321. DOI: http://dx.doi.org/10.1098/ rspb.2002.2218.

Hebert, P.D.N., Ratnasingham, S. and de Waard, J.R. 2003b. Barcoding animal life: cytochrome $c$ oxidase subunit 1 divergences among closely related species. - Proceedings of the Royal Society of London B: Biological Sciences 270: S96-S99. DOI: http://dx.doi.org/10.1098/ rsbl.2003.0025.

Kobayashi, T. 2014. A redescription of Zavrelia simantoneoa (Sasa, Suzuki and Sakai, 1998) comb. nov. - CHIRONOMUS Journal of Chironomidae Research 27: 41-44. DOI: http:// dx.doi.org/10.5324/cjcr.v0i27.1699.

Lin, X.L., Stur, E. and Ekrem, T. 2017. DNA barcodes and morphology reveal unrecognized species of Chironomidae (Diptera). - Insect Systematics \& Evolution. DOI: https://doi. org/10.1163/1876312X-00002172.

Sæther, O.A. 1969. Some Nearctic Podonominae, Diamesinae, and Orthocladiinae (Diptera: Chironomidae). - Bulletin of the Fisheries Research Board of Canada 170: 1-154.

Sæther, O.A. 1980. Glossary of chironomid morphology terminology (Diptera: Chironomidae). - Entomologica scandinavica, Supplement 14: $1-51$. 